structure, and the two heads were stacked together in $\varphi$-shaped structure. In the presence of AMPPNP, Y-shaped dynein was predominant (Y-shaped, $62.7 \%$ / $\varphi$-shaped, 24.6\%). In contrast, in an ADP-Vi condition, which mimics the ADP-Pi state of the catalytic cycle, dynein molecule tended to form a closed conformation (Y-shaped, 26.0\% / $\varphi$-shaped, 53.4\%). We constructed His-tagged recombinant human dynein heavy chains and performed a sitespecific Ni-NTA-gold labeling. Combined with single particle analysis, the orientation of the two heads in $\varphi$-shaped structure will be discussed.

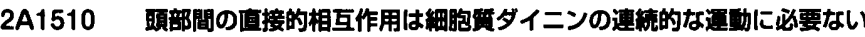

A direct interaction between dynein two heads is not necessary for processive movements of dynein

Keitaro Shibata, Yui Utsumi, Takayuki Torisawa, Yoko Toyoshima (Dept. Life Sci., Grad. Sch. Arts and Sci., Univ. Tokyo)

Cytoplasmic dynein is a two-headed motor protein which moves toward the minus end of microtubules. Recently, the crystal structure of dynein motor domain has been reported and understanding of the motor machinery has progressed. However, how dynein uses two heads during its processive movement on microtubules is not clear yet.

Previously, we reported that dynein moved on zinc-induced tubulin polymers (zinc-sheets) which include only one protofilament to be utilized by dynein movement, suggesting that dynein moves on zinc-sheets by inchworm mechanism.

Here, to elucidate whether a direct interaction between the two heads is necessary for its processive movement, we prepared an artificial dimer: the dynein motor domains are dimerized by an anti-parallel actinin rod known to have a high persistence length. EM images of this actinin-dimers revealed that the two heads keep a distance of $\sim 25 \mathrm{~nm}$ by the rigid rod. A single molecules of the actinin-dimer moved both on microtubules and on zinc-sheets processively. Our results suggest that a direct interaction between dynein two heads is not necessary for the processive movement of dynein.

\section{$2 A 1522$ 外部負荷存在下でのヒト細胞賽ダイニンのメカノケミカルサイクル}

The mechanochemical cycle of human cytoplasmic dynein under external force

Taketoshi Kambara ${ }^{1}$, Yoshiaki Tani ${ }^{1}$, Motoshi Kaya', Tomohiro Shima ${ }^{2}$, Hideo Higuchi ${ }^{1}\left({ }^{1}\right.$ Grad. Sch. Sci., Univ. Tokyo, ${ }^{2} Q B i C$, Riken)

Dynein is a molecular motor that moves toward the minus-end of microtubules. Cytoplasmic dynein play roles in positioning the Golgi complex and other organelles in cells, movement of chromosomes, and positioning the mitotic spindles during mitosis. Here, we used optical trapping to show the single molecule properties and the effect of load on the mechanochemical cycle of human cytoplasmic dynein. We expressed truncated C-terminal motor domain of dynein using baculovirus expression system. The double-headed motor domain was responsible for producing a high force of $\sim 6 \mathrm{pN}$ with a predominant step size of $8 \mathrm{~nm}$. An unbinding force of single-headed dynein was measured in various nucleotide conditions. Dynein with the biotin-tag was attached to avidin-coated polystyrene beads, and the bead was trapped by optical tweezers. The external load was imposed by moving the stage. An unbinding force measurement indicates that dynein-microtubule binding is weak for the ADP/vanadate state and strong for the nucleotide-free, AMPPNP and ADP states. The unbinding force was weaker when dynein was pushed toward the minus end of microtubule in the strongly bound state, while it was independent of loading direction in the weakly bound state. Analysis with a model revealed that the dissociation rate of dynein from microtubules increases as the force increases. Our results suggest that force plays an important role in the mechanochemical cycle of dynein to ensure the increasing of a probability for rear-head detachment with strain

\section{A1534 Rotation of microtubules driven by Tetrahymena $22 \mathrm{~S}$ outer arm dynein and its sub-particles}

Shin Yamaguchi, Yoko Toyoshima, Junichiro Yajima (Dept Life Sciences, Graduate School of Arts and Sciences, Univ. of Tokyo.)

Axonemal dynein is the molecular motor that provides motive force in cilia. Tetrahymena $22 \mathrm{~S}$ outer arm dynein, which is localized between the outerdoublet microtubules (MTs), consists of three different heavy chains, termed $\alpha$, $\beta$ and $\gamma$, and is decomposed into two-headed $\beta \gamma$ sub-particle and one-headed $\alpha$ sub-particle by chymotryptic digestion. Previous study showed that these subparticles had motility, yet it has not been clear how the three-headed structure of
$22 \mathrm{~S}$ dynein affected its force generation. To decipher the contribution of each heads to the $22 \mathrm{~S}$ dynein activity, we examined the motility of the $22 \mathrm{~S}$ dynein and its sub-particles, using three-dimensional tracking of a quantum dot attached to MTs in an in vitro motility assay. As a result, we found that surfaceattached 22S dynein and its sub-particles drove not only on-axis MT sliding, but also clockwise MT rotation around its axis. We estimated rotational pitch by fitting the $x y$-position of a quantum dot with a sine function. The pitch of $22 \mathrm{~S}$ dynein and $\beta \gamma$ sub-particle had different ATP dependency, whereas the pitch of $\alpha$ sub-particle was constant. At $10 \mu \mathrm{M}$ ATP, each dyneins showed almost same pitch. These corkscrewing motions of MTs have not been seen for both $22 \mathrm{~S}$ dynein and its sub-particles, and it demonstrates that these dyneins produce torsional forces as well as axial sliding forces. Our results suggest that torque generation by the three-headed $22 \mathrm{~S}$ dynein is not due to the cooperation between each of the three heads, but due to the intrinsic property of individual heads.

\section{$2 A 1546$ 哺釈類細胞賴ダイニンがー方向性を獲得する機棈について}

The mechanism of the transition from diffusion to directed movement in mammalian cytoplasmic dynein

Takayuki Torisawa ${ }^{1}$, Furuta Ken'ya ${ }^{2}$, Muneyoshi Ichikawa $^{1}$, Yoko Toyoshima ${ }^{1}\left({ }^{1}\right.$ Dept Life Sciences, Graduate School of Arts and Sciences, the Univ of Tokyo, ${ }^{2}$ Bio ICT lab, NICT)

Cytoplasmic dynein is a minus-end-directed molecular motor involving in various cellular functions. Mammalian cytoplasmic dynein has been reported to exhibit unidirectional movements for several micrometers in vivo (Kobayashi, 2009). However, some in vitro studies have reported that single molecules of cytoplasmic dynein/dynactin complex showed diffusive movements along microtubules (Ross, 2006; Miura, 2010), and a recent study has demonstrated that an mRNP complex bound by a few dyneins displayed diffusive movement (Amrute-Nayak, 2012). To reveal the mechanism of the transition from diffusion to directed movement in cytoplasmic dynein, we designed recombinant dyneins using HEK293 cell expressing system. An artificially dimerized, tail-truncated human cytoplasmic dynein $1(\mathrm{GST}+380 \mathrm{kD})$ showed unidirectional movement, whereas full-length dyneins purified from the same system diffused along microtubules. Furthermore, using several other recombinant dyneins that have different head-to-head distances between two motor head, we discovered that the dynein with longer head-to-head distance contained larger diffusive component. We also observed that multiple fulllength dyneins bound to Qdot moved unidirectionally along microtubules, while single dyneins carrying Qdot exhibited diffusive movement. These observations imply that the directed movement of dynein is influenced strongly by the headto-head distances and the motor number, leading to the possibility of dynein regulation by these factors in the cell.

\section{$2 A 1558$ 骨格觔形成週程における細胞筫ダイニンの分布变化}

The dynamic change of the distribution of cytoplasmic dynein during skeletal muscle differentiation process

Takuya Kobayashi, Motoshi Kaya, Hideo Higuchi (Department of physics, Graduate school of science, The university of Tokyo)

Skeletal muscles are thought to be rigid structure, but it must be always maintained. In non-muscle cells, cytoplasmic dynein is bearing important roles for intracellular maintenance. However, the roles of dynein are not understood in skeletal muscles. In order to understand the role of cytoplasmic dynein, we developed the in vivo fluorescence imaging of mouse skeletal muscle which molecular structures are visualized by using the gene transfer of GFP-fused proteins. Microtubules and cytoplasmic dyneins in in vivo muscles were successfully visualized by expressing GFP-Tubulin and dynein IC74-GFP with confocal microscope. The distribution and dynamics of vesicles transported by cytoplasmic dyneins in living muscle differed from non-muscle cells, and these vesicles did not move along the microtubule in living muscle. Meanwhile, dynein IC74-GFP were stably expressed in the myoblast cell line $\mathrm{C} 2 \mathrm{C} 12$ and, vesicle transports were observed in myoblast cell and myotube. The dynein binding vesicles in the myoblast stage distributed as similar as in non-muscle cells. However, in myotube, the vesicles mainly moved along longitudinal axis and the speed of the vesicles was decreased to $20 \%$ of myoblast stage. Thus, the distribution and dynamics of vesicle transport by cytoplasmic dyneins remarkably changed during the muscle differentiate process. 